\title{
Li-Bi-Se semiconductor thin films: technology, structure and electrophysical properties
}

\author{
V.I. Bilozertseva ${ }^{1}$, H.M. Khlyap ${ }^{2,3}$, P.S. Shkumbatyuk ${ }^{3}$, N.L. Dyakonenko ${ }^{1}$, A.O. Mamaluy ${ }^{1}$, D.O. Gaman $^{1}$ \\ ${ }^{1}$ National Technical University “KPI”, 21, Frunze str., 61002 Kharkov, Ukraine, \\ Phone:0-(57)-7076347,fax:0-(57)-7076601,e-mail: biloz@mail.ru \\ ${ }^{2}$ University of Technology, Fachbereich Physik, E.-Schrödinger-Str. 56, D-67663, Kaiserslautern, Germany, \\ ${ }^{3}$ State Pedagogical University, 24, Franko str., 82100 Drohobych,Ukraine, e-mail: hkhlyap@yahoo.com
}

\begin{abstract}
The results of structural investigations and electric field-induced properties of thin $(40-100 \mathrm{~nm}) \mathrm{Li}-\mathrm{Bi}-\mathrm{Se}$ films grown on glass substrates by means of the resistive evaporation technique are reported. The experimental investigations of microstructure and phase composition of thin films by transmission electron microscopy (TEM) and electron diffraction methods are carried out. The experimental current-voltage dependences and transport of charge carriers are discussed.
\end{abstract}

Keywords: thin film, Li-Bi-Se, structure, electron diffraction, electrical properties.

Manuscript received 06.10.09; accepted for publication 22.10.09; published online 30.12.09.

\section{Introduction}

Investigations of multicomponent condensed materials make it possible to expand their practical applications in microelectronics and sensor devices in comparison with elemental and binary materials [1-4]. Thin films of multicomponent amorphous chalcogenide semiconductors containing alkali metals enable to become a wide range of structures with unique physical and chemical properties. Bi-containing materials are seemed to be an attractive object not only for structural studies (the question is still almost open) [3], but also as a subject of electric investigations. These properties are not studied until recently.

Ternary compounds $\mathrm{A}^{\mathrm{I}} \mathrm{BiC}^{\mathrm{VI}}$ (where $\mathrm{A}^{1}=\mathrm{Li}, \mathrm{K}, \mathrm{Rb}$ and $\mathrm{C}^{\mathrm{VI}}=\mathrm{S}, \mathrm{Se}$ ) are of special importance among the chalcogenide semiconductor materials. The methods of variation of chemical composition and changing growth regimes enable to manufacture objects with different degree of disorder and various physical characteristics. Such a degree of technological freedom is principally impossible for compounds based on elemental and binary materials. Presence of alkali element improves the stability of the as-grown films that can be sensitive to different environmental conditions.

The paper describes some structural peculiarities of amorphous $\mathrm{LiBiSe}_{2}$ and polycrystalline $\mathrm{LiBi}_{3} \mathrm{Se}_{5}$ films and for the first time demonstrates their electric fieldinduced characteristics.

\section{Experimental details}

The investigated films were deposited by resistive evaporation method from quasi-closed volume under vacuum level $P=10^{-3} \mathrm{~Pa}$ on glass substrates at different substrate temperatures $T_{s}: 300$ and $400 \mathrm{~K}$. High quality powders prepared from bulk polycrystals were used as a charge. The thickness of the layers obtained was estimated to be $40-80 \mathrm{~nm}$, the rate of deposition did not exceed $0.1-0.5 \mathrm{~nm} / \mathrm{s}$. The film composition was controlled using an EMAL-2 mass analyzer with laser ion source for elemental mass spectral analysis of solids.

\section{Results and discussion}

Electron diffraction investigations demonstrated amorphous structure of the layers obtained under $T_{s}=$ $300 \mathrm{~K}$ and polycrystalline structure of the films grown at $T_{s}=400 \mathrm{~K}$. These films were also characterized by a cubic lattice of the bulk material used as a charge in the technological process.

Electron-microscopic studies exhibited "honeycomb" geometry of the film surface. Such a picture represents quasi-insulated regions with labyrinth-like contour. The relief of the investigated samples shows space density fluctuations for the amorphous films: the regions of high density (RHD) correspond to the dark network (clusters) and the regions of low density (RLD) correspond to the light network (cluster boundaries). This structure is not changed under increasing the thickness of the film. The characteristic sizes of the observed RHD are 5-20 nm. Studies of the relative size frequency distribution for $\mathrm{LiBi}_{3} \mathrm{Se}_{5}$ and $\mathrm{LiBiSe}_{2}$ films revealed that the average size of the RHD is $9.4 \mathrm{~nm}$ and this value for RLD is about $2.0 \mathrm{~nm}$.

TEM images of the as-grown layers are shown in Figs 1 and 2. Fig. 1 illustrates the structure of $\mathrm{LiBiSe}_{2}$ films. As it is seen, the contrast is represented by two of the most visible structural units: clusters $(A)$ and cluster 
boundaries $(B)$. Diffraction pattern of the investigated films indicate the amorphous structure of $\mathrm{LiBiSe}_{2}$ films (inset).

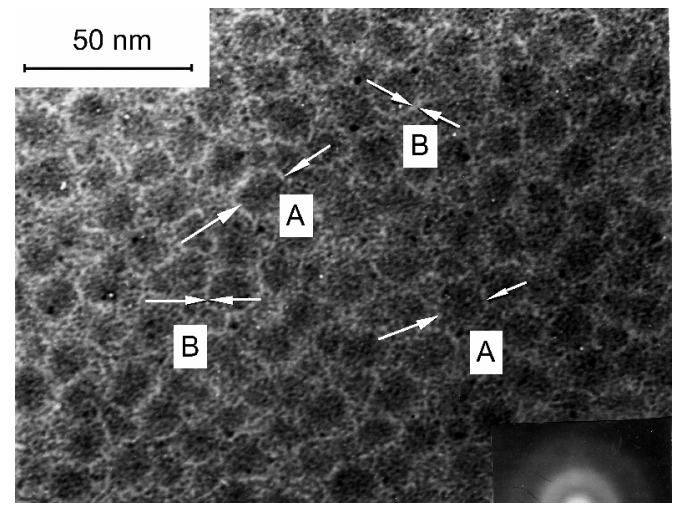

Fig. 1. TEM image of structure and electron diffraction pattern of the $\mathrm{LiBiSe}_{2}$ film with thickness of $40 \mathrm{~nm}$ deposited onto the glass substrate at the substrate temperature $T_{s}=300 \mathrm{~K}$, vacuum level $P=10^{-3} \mathrm{~Pa}$, rate of condensation $0.1-0.5 \mathrm{~nm} / \mathrm{s}$. Structural units are observed: $\mathrm{A}$ - clusters, $\mathrm{B}$ - cluster boundaries.

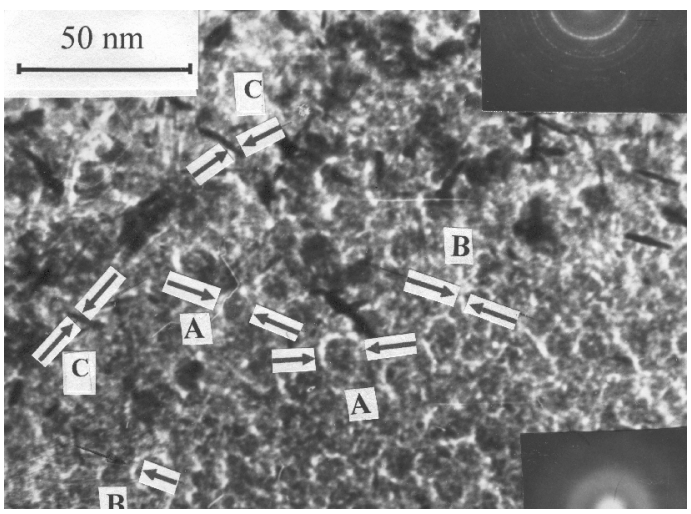

a)

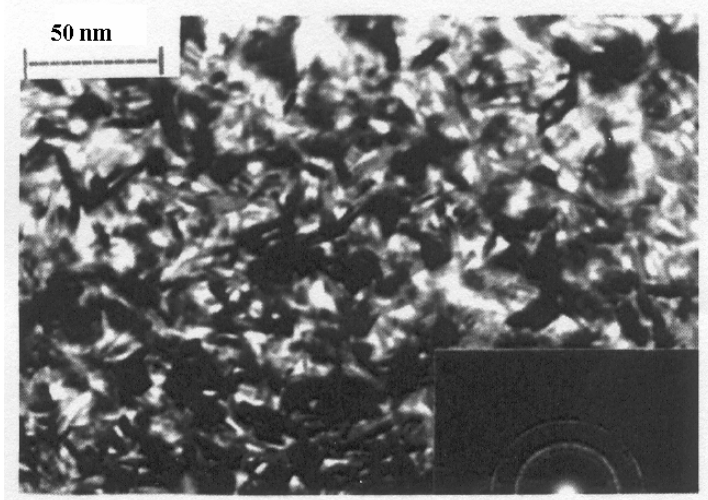

b)

Fig. 2. TEM image of structure and electron diffraction patterns of $\mathrm{LiBi}_{3} \mathrm{Se}_{5}$ films with thickness of $80 \mathrm{~nm}$ deposited onto glass substrates, vacuum level $P=10^{-3} \mathrm{~Pa}$, rate of condensation $0.1-0.5 \mathrm{~nm} / \mathrm{s}$; a) $T_{s}=300 \mathrm{~K}:$ A - clusters, B cluster boundaries, $\mathrm{C}-$ nanocrystals; b) $T_{s}=400 \mathrm{~K}: \mathrm{LiBi}_{3} \mathrm{Se}_{5}$ nanocrystals.

$\mathrm{LiBi}_{3} \mathrm{Se}_{5}$ layers have polycrystalline structure and show three types of the structural units (Fig. 2a, b): clusters $(A)$, cluster boundaries $(B)$, and nanocrystallites $(C)$. This crystals appear as a self-assembly net because of a small increase of the temperature under TEM investigations (only about $40-60 \mathrm{~K}$ ) due to electron beam focusing on the layer surface. The diffraction pattern (inset) also points out the polycrystalline structure of the film.

Electric measurements of the $\mathrm{LiBiSe}_{2}$ films were carried out at the room temperature in open air under normal atmospheric conditions. The principal attention was focused on current-voltage characteristics. Indium was used as material for contacts. Contact pads were formed by high-speed thermodeposition in order to prevent possible changes of the phase composition of the sample. The best samples with electrical area of about $3 \mathrm{~mm}^{2}$ deposited under $T_{s}=300 \mathrm{~K}$ were chosen for studies under applied bias up to $80 \mathrm{~V}$ due to the high initial resistance of the layers $\left(\sim 10^{7}-10^{8} \mathrm{Ohm}\right)$. To avoid the film heating by the applied electric field, we have restricted the bias only up to $80 \mathrm{~V}$.

The nature of electronic transport in amorphous semiconductors has attracted much attention during recent years. The electrosensitivity of the layer seemed to be important as a parameter of the material resistance. As is known [5], for low electric fields the material is in almost non-conductive state with typical values of the specific resistance within the range $10^{8}-10^{10} \mathrm{Ohm} \cdot \mathrm{cm}$ at room temperature. Under increasing applied electric field, the material is converted in a very short time from a highly resistive to a highly conductive state. Nevertheless, the question about current mechanisms is still open.

As we have mentioned above, the presented results are obtained for the first time and the experimental data are analyzed in the framework of the theory of amorphous and disordered semiconductors [6-10].

It should be noted that a contact metal-amorphous semiconductor [6] can function as a quasi-ohmic contact as well as an active element (when the barrier structure is formed). Besides, the currents due to the electron injection from the metal under applied external bias are also flowing through the contact. The possible potential barrier in this case has some important features

- the space charge region is formed under immediate participation of the charged localized states near the Fermi level $E_{F}$ in the energy range from $E_{F}$ to $E_{F}+e \phi_{0}$, where $\phi_{0}$ stands for a band bending near the semiconductor surface;

- the process of the barrier formation can be divided into two stages: i) the diffusion of free carriers during the period of about $t=10^{-12} \mathrm{~s}$ leads to the intermediate equilibrium; ii) the second stage takes place due to ionization of electrically active localized states near the Fermi level during $t \sim 10^{2}-10^{3} \mathrm{~s}$.

A very likely current mechanism in this a structure is hopping conductivity [5] related with a hopping probability $p=v_{\mathrm{ph}} \exp (-2 \alpha R) \exp \left(-W / k_{B} T\right)$, where $v_{\mathrm{ph}}$ is an attempt frequency $\left(10^{12}-10^{13} \mathrm{~s}^{-1}\right), R$ is a 
jumping distance and $\alpha$ is a quality which is representative for the rate of the fall-off of the wave function at a site or barrier. Since $R$ appears as an exponent in the tunneling factor, a small change in $R$ may cause an amplified change of the probability $p$ and, hence, of the release time. Physically it means that an injected carrier moves towards the right electrode by hopping with a constant hopping energy $W[5]$.

Experimental current-voltage characteristics of the amorphous $\mathrm{LiBiSe}_{2}$ films are plotted in Fig. 3. While there is no pre-defined polarity of the applied voltage (as in the case of heterostructures or $p-n$ junctions), we call the current flowing from the left contact to the right one as a "forward", and vice versa. The forward current slowly increases as the applied bias increases, the more considerable increase of the current is observed under reverse voltage.

It is obvious that current-voltage characteristics in both directions are very similar. The difference between the values of the currents flowing through the film in various directions may be a consequence of the sample's microstructure effect (see Fig. 1).

To analyze the experimental results, it should be taken into account that the current-voltage characteristic of a semiconductor [10] can be in principle calculated from the $j-F$ relation by integrating the current density $j$ over the cross-section $f$ of the current flow:

$I=\int j d f$,

and the field $F$ over the length $L_{z}$ of the sample:

$V=\int_{0}^{L_{z}} F(z) d z$.

This set of equations gives an applicable solution for describing the data obtained under measurements:

$I \sim V_{a}^{m}$,

where $m$ is defined only by the experiment.

Two sections observed under the applied bias can be qualitatively described as [9]:

$I \sim V_{a}^{1 / 2}$,

and the more detailed analysis led to the following expression:

$I \sim I_{s}\left(\frac{e V_{a}}{m k_{B} T}\right)^{m}$,

where $I_{s}$ takes care of a saturation current (this parameter is typical for the barrier structures and appears as a function of the carriers mobility and effective mass as well as the height of the potential barrier forming on the film surface) and the coefficient $m$ is determined experimentally. The expression for the saturation current is as follows:

$I_{s}=A^{* *} T^{2} \exp \left(\frac{-q \varphi_{B}}{k_{B} T}\right)$.
Here $\mathrm{A}^{* *}$ is the effective Richardson constant $[8,9], T$ is a temperature, $\varphi_{B}$ is a barrier height (this value is calculated using the quantum mechanic technique $[8,9]), k_{B}$ is the Boltzmann constant.

The saturation current $I_{s}$ was calculated from the semi-logarithmic experimental current-voltage characteristics rebuilt in semi-logarithmic scale $\left(\log I_{m}=f\left(V_{a}\right)\right.$, where $V_{a}$ is an applied voltage and $I_{m}$ is a measured current) as an intercept of the experimental $\log I_{m}$ curve with the ordinate axis. The corresponding numerical value was estimated to be about $10^{-10} \mathrm{~A}$ (for the "forward" direction). A small deviation from this value of "reverse" $I_{s}$ confirmed our assumption concerning the effect of the surface structure of the film on the current paths.

The calculated current-voltage characteristic is presented by the curve 3 in Fig. 3 .

As it is shown, the experimental results obtained under room-temperature electrical investigations should be interpreted within the models of the electronic structure of the material involving a proper description of electronic states. It is known ([5] and Refs therein) the potential fluctuations caused by the configurational disorder in amorphous materials may lead to the formation of localized states which do not occupy all the different energies in the band, but form tails above and below the normal band (Fig. 4).

On the basis of Davis-Mott model [5], there can be three processes leading to conduction in amorphous semiconductors. Their relative contribution to the total conductivity will predominate in different temperature regimes: i) at very low temperatures, conduction can occur by thermally assisted tunneling between states at the Fermi level; ii) at higher temperatures, charge carriers are excited into localized states of the band tails; carriers in these states can take part in the electric charge transport only by hopping; iii) at still higher temperatures, carriers are excited across the mobility edge into the extended states (it should be noted that the mobility in the extended states is much higher than in the localized states).

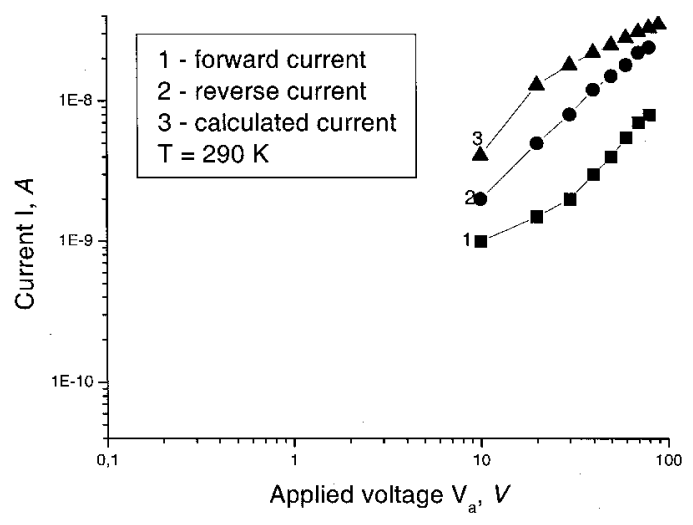

Fig. 3. Experimental (curves 1,2) and calculated (curve 3) current-voltage characteristics of investigated samples. 


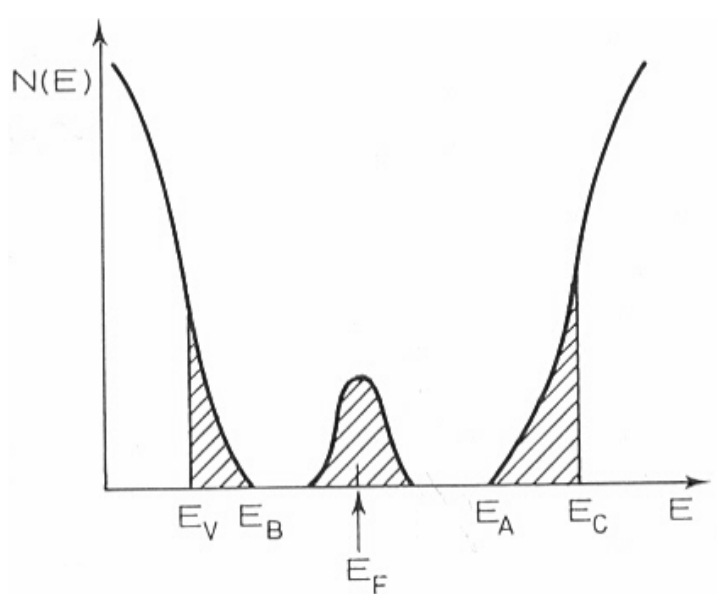

Fig. 4. Schematic image of Davis-Mott band model for chalcogenide semiconductors [5]. $E_{c}$ and $E_{v}$ represent the energies that separate the ranges where the states are localized and extended.

However, the sharp limits between these three temperature regimes are not realistic; for various chalcogenide materials they can be quite different. In our case $(T=290 \mathrm{~K})$, one can consider two competing modes of the carrier transport: tunneling and hopping. On the other hand, the measurements of current-voltage characteristics are impossible without metallic contacts. A question about the effect of metal contact (Al, Ni, Cr, In) on the properties of the investigated films remains open and disputable. As it is found for chalcogenide semiconductor materials [11], near the contacts the localized states can trap the charge of both signs, forming a space charge region and a corresponding potential barrier. Because the width of the space charge region in chalcogenide semiconductors is much smaller than that in crystalline materials [11], it also allows charge tunneling. On the other hand, a strong carrier generation [12] is a good candidate to occur due to the small gap of phase components of the film and due to the existence of slightly bonded electrons in trapped states in the amorphous chalcogenide. Therefore, we should take into account the effect of a near-contact region formed at the interface metal-film as a source emitting carriers under applied electric field.

\section{Conclusions}

Amorphous and polycrystalline Li-Bi-Se thin films of different composition have been prepared using the resistive evaporation technique from quasi-closed volume. The deposition onto glass substrates at various temperatures of the substrate $(300$ and $400 \mathrm{~K}$ ) made it possible to grow amorphous $\mathrm{LiBiSe}_{2}$ and polycrystalline $\mathrm{LiBi}_{3} \mathrm{Se}_{5}$ layers. The self-assembly growth of the needlelike nanocrystallites observed under the TEM studies of as-grown $\mathrm{LiBi}_{3} \mathrm{Se}_{5}$ layers is caused by electron beam focusing and film heating. Room-temperature currentvoltage functions of the amorphous films demonstrated the power-like dependence under forward direction of the applied voltage as well as at the reverse direction of the applied bias.

In conclusion, we can say that experimental roomtemperature current-voltage characteristics of the investigated films are mainly caused by significant decrease of the layer resistivity (and increase of the carriers mobility, respectively) under the applied electric fields in the range $\left(0-3 \times 10^{4}\right) \mathrm{V} \cdot \mathrm{m}^{-1}$.

\section{References}

1. V.I. Bilozertseva, E.O. Lupashko, V.V. Mussil, A.P. Ovcharenko, Multilayer photorecording medium and devices for recording and processing of information // Proc. SPIE 2648, p. 688-693 (1995).

2. V.I. Bilozertseva, V.A. Bazakutsa, L.N. Panchenko, Structure and crystallization of $\mathrm{Ag}_{3} \mathrm{SbS}_{3}$ layers obtained by pulse laser evaporation // Cryst. Res. Techn. 5-6, p. 677 (1999).

3. V.A. Bazakutsa, A.A. Ryabchun, N.L. Dyakonenko, I.V. Shelest, V.I. Bilozertseva, Columnar structure of $\mathrm{A}^{\mathrm{I}} \mathrm{Bi}_{3} \mathrm{C}_{5}{ }^{\mathrm{VI}}$ amorphous films // Functional Materials 6, No. 1, p. 151 (1999).

4. V.I. Bilozertseva, V.A. Bazakutsa, S.D. Gapochenko, Ye.T. Lemeshevskaya, V.V. Mussil, Optical properties and photo-induced effects in GeAs-Se films and two-layer systems on their basis // Proc. SPIE, 5024, p. 80-84 (2003).

5. P. Nagels, Electronic transport in amorphous semiconductors, in: M. H. Brodsky (Ed.), Amorphous Semiconductors, $2^{\text {nd }}$ ed., Ch. 5, p. 113158. Springer, Berlin, 1985.

6. N. Vishnyakov, S. Vikhrov, V. Mishustin et al. Potential barriers formation in undoped disordered semiconductors // Fizika tekn. Poluprov. 39 (10), p. 1189 (2005), in Russian.

7. V. Bonch-Bruevich and R. Enderlein (Eds.), Electronic Theory of Disordered Semiconductors. Nauka Publishers, Moscow, 1981.

8. E. Schöll, Non-Equilibrium Phase Transitions in Semiconductors, p. 63. Berlin, Springer, 1987.

9. S.M. Sze, K. Ng Kwok, Physics of Semiconductor Devices, $3^{\text {rd }}$ Edition, Ch. 3, p. 134-197. Wiley, New York, 2007.

10. B. Braunecker, D. Feldman and J. Marston, Rectification in one-dimensional electronic systems // Phys. Rev. B72, 125311 (2005).

11. D. Tsiulyanu, Heterostructures on chalcogenide glass and their applications, in: Semiconducting Chalcogenide Glass III, Eds. R. Fairman, B. Ushkov. Elsevier, Academic Press, p. 15-46 (2004).

12. P. Fantini, A. Pirovano, D. Ventrice and A. Redaelli, Experimental investigation of transport properties in chalcogenide materials through $I / f$ noise measurements // Appl. Phys. Lett. 88, 263506-1-263506-3 (2006). 